\title{
Monogenic pseudo-complex power functions and their applications
}

\author{
Carla Cruz ${ }^{a}$ \\ M.I. Falcão ${ }^{b}$ \\ H.R. Malonek ${ }^{a}$ \\ ${ }^{a}$ Departamento de Matemática, Universidade de Aveiro, Portugal \\ ${ }^{b}$ Departamento de Matemática e Aplicações, Universidade do Minho, Portugal
}

\begin{abstract}
The use of a non-commutative algebra in hypercomplex function theory requires a large variety of different representations of polynomials suitably adapted to the solution of different concrete problems. Naturally arises the question of their relationships and the advantages or disadvantages of different types of polynomials. In this sense, the present paper investigates the intrinsic relationship between two different types of monogenic Appell polynomials. Several authors payed attention to the construction of complete sets of monogenic Appell polynomials, orthogonal with respect to a certain inner product, and used them advantageously for the study of problems in 3D-elasticity and other problems. Our goal is to show that, as consequence of the binomial nature of those generalized Appell polynomials, their inner structure is determined by interesting combinatorial relations in which the central binomial coefficients play a special role. As a byproduct of own interest, a Riordan-Sofo type binomial identity is also proved.
\end{abstract}

\section{Information}

Keywords:

Functions of hypercomplex variables; combinatorial identities; generalized Appell polynomials.

Original publication:

Mathematical Methods in Applied Sciences 37, (2014), 17231735

DOI: $10.1002 / \mathrm{mma} .2931$

http://onlinelibrary.wiley.com

\section{Introduction}

It is a well known fact that the study of solutions of partial differential equations, particularly of CauchyRiemann type with well adapted algebraic methods can lead to new and important insights in their structure. Weierstrass noticed in a letter to H. A. Schwarz, October 3, 1875, [1], that

The more I ponder the principles of function theory - and I do so unceasingly - the firmer becomes my conviction that they have to be built on a foundation of algebraic truths.

Indeed, a natural approach to the treatment of problems in an even number of real variables greater than two, is the application of several complex variables. This was already done by Weierstrass himself. But immediately arises the question of how to deal in a similar way with problems, if the number of real variables is odd. In the work of Moisil and Théodoresco [2] we can find the first example of a systematic approach related to vector functions of three real variables and relying on the algebra of $3 \times 3$ matrices.

At almost the same time, the article [3] of the Swiss mathematician R. Fueter, being a disciple of D. Hilbert and well known number theorist, showed also the relevance of the algebra of quaternions in this context. His advances in direction of a hypercomplex function theory based on quaternions as a generalization of complex numbers, revealed already the fact that in both cases, i.e. in the case of an odd as well as of an even number of real variables, the non-commutative algebra of quaternions can advantageously be used. Remarkable is also the fact that in the last version of his lectures on function theory [4] he already used Clifford Algebras over 
$n+1$ real variables, namely for the representation of the solution of the Dirac equation in higher dimensions as a generalization of Cauchy-Riemann equations in complex analysis.

Dormant for almost twenty years, R. Delanghe [5] restarted in the beginning of the 70-ies in the spirit of Fueter a systematic development of what is now called Clifford Analysis. Since that time we can find a huge number of theoretical as well as application oriented papers published in this field. In what concerns the representation of polynomials in several real variables by application of Clifford Algebras, different techniques have been developed. As examples we mention here the use of the Fischer decomposition for harmonic polynomials as well as the Cauchy-Kovalevskaya extension for real analytic Clifford Algebra valued functions of $n$-real variables to those of monogenic functions in $n+1$ variables. Both approaches have been described in extension in [6]. Also the use of Legendre or Gegenbauer polynomials as starting point is very common (see $[7,8])$. The diversity of polynomial representations can be explained with the fact that composition and multiplication are not closed operations in the set of generalized holomorphic (monogenic) functions. Therefore different problems frequently demand different representations of polynomials (cf. [9]).

This became particular evident when two of the authors of this paper solved the question of existence of homogeneous monogenic polynomials possessing the property of a generalized Appell sequence with respect to a hypercomplex derivative $([10,11])$. Meanwhile this approach to power-like functions in several hypercomplex variables has attracted the attention of various authors. It has been considered from different points of view, including approaches based on representation theoretic methods $[12,13]$ and in connection with the direct construction of orthogonal polynomial sequences [14]. The construction of an orthogonal bases as it is described in $[12,13]$ (and others), relies on general properties of Gelfand-Tsetlin bases. The approach in [14] plays in a very clever way with properties of the so-called generalized monogenic constants.

In this paper we look for the intrinsic relationship between a particular sequence of monogenic Appell polynomials in those complete sets of orthogonal monogenic Appell polynomials and another type of generalized Appell polynomials which are characterized by the property of being isomorphic to the integer complex powers $z^{k}, z \in \mathbb{C}, k=0,1, \ldots$, for arbitrary dimensions $n$. They have been used for the first time in approximation problems [15] and later on they appeared as examples of Appell polynomials written in terms of several hypercomplex variables in [16]. The fact that they form a $(n-1)$-parameter family of generalized holomorphic Appell sequences allows also to construct complete sets of homogeneous power-like functions of degree $k$ by variation of the parameters. For simplicity we will concentrate on the simplest case of an odd number of real variables, i.e. the case of $n+1=3$, which corresponds to the case of two hypercomplex variables depending on one real parameter. In this case, the investigation of the relationship between the Appell sequence considered in [15] (which is contained in the complete sets of orthogonal monogenic Appell polynomials considered in [12] and [14]) and those monogenic power functions reveals an amazing connection with the central binomial coefficients not studied so far.

We recall that in enumerative combinatorics, the sequence of Catalan numbers $\mathcal{C}_{m}$ defined as weighted central binomial coefficients in the form

$$
\mathcal{C}_{m}=\frac{1}{m+1}\left(\begin{array}{c}
2 m \\
m
\end{array}\right), \quad \text { for } \quad m \geq 0
$$

occurs in many counting problems, interesting combinatorial identities, as well as in several important applications in number theory and other fields. The use of the weight $\frac{1}{2^{2 m}}$ instead of $\frac{1}{m+1}$ leads to another interesting sequence of numbers constructed with the help of the central binomial coefficient, namely

$$
c_{2 m}=\frac{1}{2^{2 m}}\left(\begin{array}{c}
2 m \\
m
\end{array}\right)=\frac{1}{2^{2 m-1}}\left(\begin{array}{c}
2 m-1 \\
m-1
\end{array}\right), \quad \text { for } \quad m \geq 0 .
$$

Relation (1) shows that in the case of this weight, it makes sense to combine the designation of both terms in (1), by using the so-called generalized central binomial coefficient $\left(\begin{array}{c}k \\ \left\lfloor\frac{k}{2}\right\rfloor\end{array}\right)$ and defining

$$
c_{k}:=\frac{1}{2^{k}}\left(\begin{array}{c}
k \\
\left\lfloor\frac{k}{2}\right\rfloor
\end{array}\right)
$$

where $\lfloor$.$\rfloor is the usual floor function and k \geq 0$. At the same time $(1)$ shows that the sequence $\left(c_{k}\right)_{k \geq 0}$ is completely defined by the ordinary central binomial coefficients $\left(\begin{array}{c}2 m \\ m\end{array}\right)$, since $c_{2 m-1}=c_{2 m}$. It seems to us 
an interesting fact that in an huge number of combinatorial identities involving the sequence of the central binomial coefficients, they occur just in the form of $\left(c_{k}\right)_{k \geq 0}$ (see, for example, $\left.[17,18,19,20]\right)$.

One of those central binomial coefficient identities, which has resisted for many years a typical combinatorial proof by "two ways of counting", is the famous Reed-Dawson Identity, first mentioned and proved in 1968 by using a recurrence relation (see [20]; also mentioned as Knuth's Old Sum in [21]):

$$
\sum_{k=0}^{2 m} \frac{(-1)^{k}}{2^{k}}\left(\begin{array}{c}
2 k \\
k
\end{array}\right)\left(\begin{array}{c}
2 m \\
k
\end{array}\right)=\frac{1}{2^{2 m}}\left(\begin{array}{c}
2 m \\
m
\end{array}\right)=c_{2 m}
$$

In the case of an odd upper limit the sum simply vanishes, i.e.

$$
\sum_{k=0}^{2 m+1} \frac{(-1)^{k}}{2^{k}}\left(\begin{array}{c}
2 k \\
k
\end{array}\right)\left(\begin{array}{c}
2 m+1 \\
k
\end{array}\right)=0 .
$$

Another example is the Riordan-Sofo Identity, proved in [18] together with one more proof of the Reed-Dawson Identity and closely related to it:

$$
\sum_{u=0}^{m} \frac{(-1)^{u}}{2^{2 u}}\left(\begin{array}{c}
2 u \\
u
\end{array}\right)\left(\begin{array}{c}
m \\
u
\end{array}\right)=\sum_{u=0}^{m}(-1)^{u}\left(\begin{array}{c}
m \\
u
\end{array}\right) c_{2 u}=\sum_{u=0}^{m}(-1)^{m-u}\left(\begin{array}{c}
m \\
u
\end{array}\right) c_{2 m-2 u}=c_{2 m} .
$$

In this paper we will show that the sequence (2) and a generalization of the Riordan-Sofo Identity (3) play a significant role in the context of hypercomplex function theory. As could been shown for the first time in [10], the explicit representation of a sequence of hypercomplex generalized Appell polynomials is essentially determined by those $c_{k}$.

The paper is organized in the following way. Section 2 introduces the necessary fundamental definitions of Hypercomplex Function Theory needed for use in the subsequent sections. Section 3 is dedicated to properties and relations of generalized Appell polynomials of two hypercomplex variables. In particular, two different types of this class of polynomials with binomial expansion are presented, which have been recently proved to play an important role in Hypercomplex Function Theory [22].

Section 4 starts with two auxiliary results of own interest. The first fills in a gap in the explicit expression of the coefficients of the standard Appell polynomials in terms of two hypercomplex variables, while the second one presents auxiliary relations deduced from properties of primitive roots of unity.

The subsequent proof of a binomial identity is the last step to the main result. As already mentioned before, it is the proof of a generalization of a result by A. Sofo in [18] with a strong relationship to the Reed-Dawson Identity. Indeed, both identities are two special cases of a generalized binomial sum studied by A. Sofo in [18].

Theorem 3 at the end of Section 4 can be considered as the main result, showing the importance of combinatorics in hypercomplex function theory and vice verse, i.e. the possibility of using hypercomplex function theory as an analytic tool for obtaining new combinatorial identities.

\section{Hypercomplex Function Theory toolbox}

Let $\left\{1, e_{1}, e_{2}, e_{3}\right\}$ be an orthonormal basis of the Euclidean vector space $\mathbb{R}^{4}$ with the (quaternionic) product given according to the multiplication rules

$$
e_{1}^{2}=e_{2}^{2}=e_{3}^{2}=-1, \quad e_{1} e_{2}=-e_{2} e_{1}=e_{3}
$$

Considering the subset $\mathcal{A}:=\operatorname{span}_{\mathbb{R}}\left\{1, e_{1}, e_{2}\right\}$ of the quaternion algebra $\mathbb{H}$ (isomorphic to the special Clifford Algebra $\left.\mathcal{C} \ell_{0,2}\right)$, the real vector space $\mathbb{R}^{3}$ can be embedded in $\mathcal{A}$ by the identification of each element $\left(x_{0}, x_{1}, x_{2}\right) \in \mathbb{R}^{3}$ with the paravector

$$
x=x_{0}+x_{1} e_{1}+x_{2} e_{2} \in \mathcal{A}
$$


Here $x_{0}=\operatorname{Sc}(x)$ and $\underline{x}=\operatorname{Vec}(x)=e_{1} x_{1}+e_{2} x_{2}$ are the so-called scalar and vector part of the paravector $x \in \mathcal{A}$, respectively. The conjugate of $x$ is given by $\bar{x}=x_{0}-\underline{x}$ and its norm by $|x|=\sqrt{x \bar{x}}$. Observe that $\underline{x}^{2}$ is a nonpositive real number and we can write $|x|^{2}=x_{0}^{2}+x_{1}^{2}+x_{2}^{2}=x_{0}^{2}-\underline{x}^{2}$.

To call attention to its relation to the complex Wirtinger derivatives, we use for a generalized CauchyRiemann operator in $\mathbb{R}^{3}$ the notation

$$
\bar{\partial}:=\frac{1}{2}\left(\partial_{0}+\partial_{\underline{x}}\right), \quad \partial_{0}:=\frac{\partial}{\partial x_{0}}, \quad \partial_{\underline{x}}:=e_{1} \frac{\partial}{\partial x_{1}}+e_{2} \frac{\partial}{\partial x_{2}} .
$$

$\mathcal{C}^{1}(\Omega, \mathcal{A})$-functions $f$, defined in a domain $\Omega$, satisfying the equation $\bar{\partial} f=0$ (resp. $f \bar{\partial}=0$ ) are called left monogenic (resp. right monogenic). We suppose that $f$ is hypercomplex differentiable in $\Omega$ in the sense of [23] and [24], i.e. has a uniquely defined areolar derivative $f^{\prime}$ in each point of $\Omega$. Then $f$ is real differentiable and $f^{\prime}$ can be expressed by real partial derivatives as $f^{\prime}=\partial f$ where analogously to the generalized CauchyRiemann operator we use $\partial:=\frac{1}{2}\left(\partial_{0}-\partial_{\underline{x}}\right)$ for the conjugate Cauchy-Riemann operator. Since a hypercomplex differentiable function belongs to the kernel of $\bar{\partial}$, it follows that in fact $f^{\prime}=\partial_{0} f=-\partial_{\underline{x}} f$ similar to the complex case.

Using the general approach to Clifford Algebra valued monogenic functions $([8,24])$ restricted to our case of $n=2$, a second hypercomplex structure of $\mathbb{R}^{3}$, different from the one given by the set of paravectors $\mathcal{A}$, consists in the following isomorphism:

$$
\mathbb{R}^{3} \cong \mathcal{H}^{2}=\left\{\left(z_{1}, z_{2}\right): z_{k}=x_{k}-x_{0} e_{k} ; x_{0}, x_{k} \in \mathbb{R}, k=1,2\right\} .
$$

Throughout this paper we consider $\mathcal{A}$-valued monogenic functions $f$ of the form

$$
f\left(z_{1}, z_{2}\right)=f\left(x_{0}, x_{1}, x_{2}\right)=f_{0}\left(x_{0}, x_{1}, x_{2}\right)+f_{1}\left(x_{0}, x_{1}, x_{2}\right) e_{1}+f_{2}\left(x_{0}, x_{1}, x_{2}\right) e_{2},
$$

where $f_{k}, k=0,1,2$ are real valued functions in a domain $\Omega \subset \mathbb{R}^{3}$. We point out that in such a case a left monogenic function is also right monogenic.

We can now introduce the notion of generalized power, an important tool in this work.

Definition 1 Let $V_{+}$, be a ring and $v_{k} \in V(k=1, \ldots, n)$. The symmetric " $\times$ "-product is defined by

$$
v_{1} \times v_{2} \times \cdots \times v_{n}=\frac{1}{n !} \sum_{\pi\left(k_{1}, \ldots, k_{n}\right)} v_{k_{1}} v_{k_{2}} \cdots v_{k_{n}},
$$

where the sum runs over all permutations of all $\left(k_{1}, \ldots, k_{n}\right)$.

Definition 2 The function $z_{1}{ }^{k-s} \times z_{2}{ }^{s}$, for $k=0,1, \ldots, s=0,1, \ldots, k$, where by convention

$$
z_{1}^{k-s} \times z_{2}^{s}=\underbrace{z_{1} \times \cdots \times z_{1}}_{k-s} \times \underbrace{z_{2} \times \cdots \times z_{2}}_{s},
$$

is called a generalized power of degree $k$.

The generalized powers defined by (4) have important properties, namely they are homogeneous monogenic polynomials with values in $\mathcal{A}$ (see [25]). Moreover, an integer power of the variables $z_{1}$ and $z_{2}$ is a monogenic function. A variable with such a property is called, in [5], a totally regular variable.

In addition, applying convention (4), the following binomial formula (see, e.g. [24]) can be derived:

$$
\left(\alpha z_{1}+\beta z_{2}\right)^{k}=\sum_{s=0}^{k}\left(\begin{array}{l}
k \\
s
\end{array}\right) \alpha^{k-s} \beta^{s} z_{1}^{k-s} \times z_{2}^{s}, \quad \text { with } \quad \alpha, \beta \in \mathbb{R} .
$$

The description of the series development of monogenic functions is made here in terms of the totally regular variables $z_{1}$ and $z_{2}$. In [25] it is proved that the set of all the $k+1$ generalized powers of degree $k=0,1, \ldots$ defined by (4) forms a basis for the Taylor series of a monogenic function in $\mathbb{R}^{3}$. More precisely, we have

$$
h\left(z_{1}, z_{2}\right)=\sum_{k=0}^{\infty} \frac{1}{k !} \sum_{s=0}^{k}\left(\begin{array}{l}
k \\
s
\end{array}\right) \frac{\partial^{k} h(0)}{\partial x_{1}^{k-s} \partial x_{2}^{s}} z_{1}^{k-s} \times z_{2}^{s},
$$


where $h$ is defined in the bicylinder $V=\left\{\left(z_{1}, z_{2}\right) \in \mathcal{H}^{2}:\left|z_{j}\right|<r_{j}, r_{j}>0, j=1,2\right\}$.

On the other hand, we recall that the Taylor series expansion of an analytic function of two complex variables $\left(w_{1}, w_{2}\right) \in \mathbb{C}^{2}$ is $([26])$

$$
f\left(w_{1}, w_{2}\right)=\sum_{k=0}^{\infty} \frac{1}{k !} \sum_{s=0}^{k}\left(\begin{array}{l}
k \\
s
\end{array}\right) \frac{\partial^{k} f(0)}{\partial w_{1}^{k-s} \partial w_{2}^{s}} w_{1}^{k-s} w_{2}^{s},
$$

where $f$ is defined in the bicylinder $U=\left\{\left(w_{1}, w_{2}\right) \in \mathbb{C}^{2}:\left|w_{j}\right|<r_{j}, r_{j}>0, j=1,2\right\}$.

Both expansions (6) and (7) show the deep analogy between functions of two hypercomplex variables and of two complex variables. The only one formal difference of both Taylor series expansions is the use of the symmetric product instead of the ordinary complex product. Notice that the convergence domain of (6) is the intersection of two cylinders in $\mathbb{R}^{3}$ and therefore allows a direct visualization, which is not the case of functions defined in $\mathbb{C}^{2}$.

\section{Appell polynomials in $\mathbb{R}^{3}$}

Some years ago, two of the authors constructed special homogeneous monogenic polynomials with the behavior of power-like functions under hypercomplex differentiation ([10,11]). More precisely, based on Appell's concept (cf. [27]) the following definition was introduced:

Definition 3 A sequence of homogeneous monogenic polynomials $\left(\mathcal{Q}_{k}\right)_{k \geq 0}$ of degree $k$ is called a generalized Appell sequence with respect to $\partial$ if

1. $\mathcal{Q}_{0}(x) \equiv 1$,

2. $\partial \mathcal{Q}_{k}=k \mathcal{Q}_{k-1}, \quad k=1,2, \ldots$

Based on this definition, the following $\mathcal{A}$-valued generalized Appell polynomials, expressed in terms of the totally regular variables $z_{1}$ and $z_{2}$, were obtained:

$$
\boldsymbol{P}_{k}\left(z_{1}, z_{2}\right):=c_{k} \sum_{s=0}^{k} z_{1}^{k-s} \times z_{2}^{s}\left(\begin{array}{l}
k \\
s
\end{array}\right) e_{1}^{k-s} \times e_{2}^{s},
$$

where the coefficients $c_{k}$ are the weighted central binomial coefficients defined by (2). To be brief, we call here these homogeneous Appell polynomials the standard Appell polynomials, since they appear also in a natural way in the set of orthogonal Appell polynomials constructed in [12] and [14].

Of special interest for the following is now a remarkable property of the first degree standard Appell polynomial, as one of the two basis polynomials, necessary for the representation of any arbitrary monogenic polynomial of degree one ${ }^{1}$.

$$
\boldsymbol{P}_{1}\left(z_{1}, z_{2}\right):=\frac{1}{2}\left(z_{1} e_{1}+z_{2} e_{2}\right)=x_{0}+\frac{1}{2}\left(x_{1} e_{1}+x_{2} e_{2}\right)
$$

The possibility of decomposing it additively in the form

$$
\boldsymbol{P}_{1}\left(z_{1}, z_{2}\right):=\frac{1}{2}\left(z_{1} e_{1}+z_{2} e_{2}\right)=\frac{1}{2}\left[\frac{\left(z_{1}+z_{2}\right)}{\sqrt{2}} \frac{\left(e_{1}+e_{2}\right)}{\sqrt{2}}+\frac{\left(z_{1}-z_{2}\right)}{\sqrt{2}} \frac{\left(e_{1}-e_{2}\right)}{\sqrt{2}}\right]
$$

leads to two different and linearly independent first degree monogenic polynomials of the form

$$
\boldsymbol{R}\left(z_{1}, z_{2}\right):=\left(z_{1} i_{1}+z_{2} i_{2}\right)\left(i_{1} e_{1}+i_{2} e_{2}\right)
$$

where $\left(i_{1}, i_{2}\right)=\left(\frac{1}{\sqrt{2}}, \frac{1}{\sqrt{2}}\right)$, respectively $\left(i_{1}, i_{2}\right)=\left(\frac{1}{\sqrt{2}},-\frac{1}{\sqrt{2}}\right)$, are unit vectors of real parameters. This can be easily verified by inspection.

\footnotetext{
${ }^{1}$ The dimension of the space of homogeneous polynomials of degree $k$ in $\mathbb{R}^{n+1}$ is equal to $\left(\begin{array}{c}n+k-1 \\ k\end{array}\right)$, cf. [8].
} 
The same reasoning can be applied in the case of $\mathbb{R}^{n+1}$ for arbitrary $n \geq 2$ where the corresponding first degree standard Appell polynomial is given by

$$
\boldsymbol{P}_{1}^{(n)}\left(z_{1}, \ldots, z_{n}\right):=\frac{1}{n}\left(z_{1} e_{1}+\ldots+z_{n} e_{n}\right)=x_{0}+\frac{1}{n}\left(x_{1} e_{1}+\ldots+x_{n} e_{n}\right) .
$$

In this general case an analogously additive decomposition of $\boldsymbol{P}_{1}^{(n)}$ leads to $2^{n-1}$ summands of the form

$$
\boldsymbol{R}^{(\boldsymbol{n})}\left(z_{1}, \ldots, z_{n}\right):=\left(z_{1} i_{1}+\ldots+z_{n} i_{n}\right)\left(i_{1} e_{1}+\ldots+i_{n} e_{n}\right)
$$

where $\left(i_{1}, \ldots, i_{n}\right)$ are the corresponding unit vectors of $n$ real parameters. A difference to the case $n=2$ consists in the following. Whereas for $n=2$ the decomposition process results in the number of two basis polynomials just needed, we have for $n \geq 3$ that $n<2^{n-1}$ and the resulting number $2^{n-1}$ of basis polynomials is bigger than the dimension and therefore they cannot anymore be linear independent. Of course, this is a minor problem and can be solved by a proper choice of the linear independent set of basis polynomials for every degree $k=1,2, \ldots$

We mentioned here the described procedure as a way of deriving from the structure of the standard Appell polynomials by decomposition a connection to a simple general set of homogeneous polynomials of first degree.

Moreover, recently in [22], it has been proved that beyond (8) there is just one type of Appell polynomials with values in $\mathcal{A}$, based on the totally regular variable (11), namely,

$$
\begin{aligned}
\mathcal{R}_{\boldsymbol{i}}^{k}\left(z_{1}, z_{2}\right) & =\left(z_{1} i_{1}+z_{2} i_{2}\right)^{k}\left(i_{1} e_{1}+i_{2} e_{2}\right)^{k} \\
& =\left[x_{0}+\left(i_{1} e_{1}+i_{2} e_{2}\right)\left(i_{1} x_{1}+i_{2} x_{2}\right)\right]^{k},
\end{aligned}
$$

where $\boldsymbol{i}:=\left(i_{1}, i_{2}\right)$ is a unit vector of real parameters and $k=0,1, \ldots$

It is easy to recognize in expression (14b) the isomorphism between these polynomials and the complex powers $z=(x+i y)^{k}$. This observation suggests the designation of (14a) or (14b) as pseudo-complex powers. Most striking is the fact that with these type of Appell polynomials we have for every value of $k$ and any number $n$ of hypercomplex variables one and the same structure of basis polynomials which allows, in the hypercomplex case, a new series representation. ${ }^{2}$ It means that we can consider homogeneous monogenic polynomials of the form

$$
\boldsymbol{S}_{\boldsymbol{k}}^{(\boldsymbol{n})}\left(z_{1}, z_{2}\right)=\sum_{l=0}^{k} \alpha_{i_{l}}^{k} \mathcal{R}_{i_{l}}^{k}\left(z_{1}, z_{2}\right),
$$

with coefficients $\alpha_{i_{l}}^{k} \in \mathcal{A}$ depending on the chosen parameter set $\left\{i_{l}=\left(i_{1 l}, i_{2 l}\right), l=0,1, \ldots k\right\}$. What concerns a difference to the one complex variable case is the fact that the $m$-fold hypercomplex derivative applied to all the elements of a pseudo-complex power basis for homogeneous polynomials of degree $k$ yields only a system of not linear independent pseudo-complex powers of degree $(k-m)$, i. e. does not reproduce a basis, since there is no reduction of the overall quantity of basis elements to the necessary number for degree $(k-m)$. In the one complex variable case the $m$-fold complex derivative reproduces again a basis element.

Notice now that (14a) is the $n=2$ case of the Appell polynomials used in [16]. There, one can find the following result concerning the use of pseudo-complex powers as a basis of homogeneous monogenic paravector valued polynomials different from $\left\{z_{1}^{k-s} \times z_{2}^{s}\right\}_{s=0}^{k}$.

Theorem 1 The set of polynomials $\left\{\mathcal{R}_{\boldsymbol{i}_{s}^{k}}^{k}\right\}_{s=0}^{k}$ of the form (14) is a basis for the space of homogeneous monogenic paravector valued polynomials of degree $k$ in $\mathbb{R}^{3}$, provided that the $k+1$ unit vectors $i_{s}^{k}=$ $\left(i_{s 1}^{k}, i_{s 2}^{k}\right) \in \mathbb{R}^{2}$, with $s=0, \ldots, k$, are pairwise noncollinear.

The importance of Appell sequences in hypercomplex context together with the fact that the polynomials (8) show interesting properties, motivated us to ask for the bijection between the representation (8) of $\boldsymbol{P}_{k}$ and its representation in terms of elements of a particular basis of the form

$$
\left\{\mathcal{R}_{\boldsymbol{i}_{0}^{k}}^{k}, \mathcal{R}_{\boldsymbol{i}_{1}^{k}}^{k}, \ldots, \mathcal{R}_{\boldsymbol{i}_{k-1}^{k}}^{k}, \mathcal{R}_{\boldsymbol{i}_{k}^{k}}^{k}\right\}
$$

\footnotetext{
${ }^{2}$ Compare (6) and (7) where the strong similarity of both Taylor series expansions in $\mathbb{C}^{2}$ for two complex variables, resp. in $\mathcal{H}^{2}$ for two hypercomplex variables, is mentioned.
} 
It is clear that the conditions referred in Theorem 1 still allow a wide choice of parameter-vectors, leading to different sets in (16). In fact, each unit vector $i_{s}^{k}$ can be written in the form

$$
\boldsymbol{i}_{s}^{k}=\left(\cos \alpha_{s}^{k}, \sin \alpha_{s}^{k}\right),
$$

where $\alpha_{s}^{k}$ are real numbers. It is clear that the parameter set $\boldsymbol{A}^{k}=\left\{\alpha_{0}^{k}, \alpha_{1}^{k}, \ldots, \alpha_{k}^{k}\right\}$ defines completely the set of unit vectors $\left\{\boldsymbol{i}_{0}^{k}, \boldsymbol{i}_{1}^{k}, \ldots, \boldsymbol{i}_{k}^{k}\right\}$.

We use throughout this work the parameter set

$$
\boldsymbol{A}^{k}=\left\{\alpha_{0}^{k}, \alpha_{1}^{k}, \ldots, \alpha_{k}^{k}\right\},
$$

with

$$
\begin{aligned}
& \alpha_{0}^{k}=\alpha_{0}^{0}=\frac{\pi}{2}, \\
& \alpha_{s}^{k}=\alpha_{s}^{s}=\frac{\pi}{2}-\frac{\left(2 n^{\prime}+1\right) \pi}{2^{n}}, \text { for } s=1, \ldots, k,
\end{aligned}
$$

where $n$ and $n^{\prime}$ are integers such that $s=2^{n-1}+n^{\prime}$, for $0 \leq n^{\prime} \leq 2^{n-1}-1$. According to Theorem 1 , this parameter set defines a basis for the space of homogeneous monogenic paravector valued polynomials $\left\{\mathcal{R}_{\boldsymbol{i}_{s}^{k}}^{k}\right\}_{s=0}^{k}$ of degree $k$, since the corresponding $k+1$ unit vectors $\boldsymbol{i}_{s}^{k}, s=0, \ldots, k$ are obviously pairwise noncollinear.

Remark 1 It is clear that (17) defines a family of sets satisfying the condition $\boldsymbol{A}^{k-1} \subset \boldsymbol{A}^{k}$, i.e. $\boldsymbol{A}^{k}=$ $\boldsymbol{A}^{k-1} \cup\left\{\alpha_{k}^{k}\right\}$. Moreover these sets (17) can be written explicitly as

$$
\boldsymbol{A}^{k}=\left\{\frac{l \pi}{2^{n-1}}\right\}_{l=-2^{n-2}+1}^{2^{n-2}} \cup\left\{\frac{\pi}{2}-\frac{(2 l+1) \pi}{2^{n}}\right\}_{l=0}^{n^{\prime}} .
$$

The last expression reveals a block structure of length $2^{n-1}$. Each block starts with $n^{\prime}=0$, i.e.

$$
\boldsymbol{A}^{2^{n-1}}=\left\{\frac{l \pi}{2^{n-1}}\right\}_{l=-2^{n-2}+1}^{2^{n-2}} \cup\left\{\frac{\pi}{2}-\frac{\pi}{2^{n}}\right\}
$$

and ends with $n^{\prime}=2^{n-1}-1$, where

$$
\boldsymbol{A}^{2^{n}-1}=\left\{\frac{2 l \pi}{2^{n}}\right\}_{l=-2^{n-2}+1}^{2^{n-2}} \cup\left\{\frac{(2 l+1) \pi}{2^{n}}\right\}_{l=-2^{n-2}}^{2^{n-2}-1}=\left\{\frac{l \pi}{2^{n}}\right\}_{l=-2^{n-1}+1}^{2^{n-1}} .
$$

From now on we denote, for simplicity, the pseudo-complex powers associated with the choice (17) by

$$
R_{s}^{k}=\left(z_{1} \cos \left(\alpha_{s}^{k}\right)+z_{2} \sin \left(\alpha_{s}^{k}\right)\right)^{k}\left(\cos \left(\alpha_{s}^{k}\right) e_{1}+\sin \left(\alpha_{s}^{k}\right) e_{2}\right)^{k} .
$$

Table 1 illustrates the structure of the parameter set (17), while Table 2 presents the first pseudo-complex powers $R_{s}^{k}$.

\begin{tabular}{ccl|ccl}
$k$ & $\alpha_{k}^{k}$ & $\boldsymbol{A}^{k}$ & $k$ & $\alpha_{k}^{k}$ & $\boldsymbol{A}^{k}$ \\
\hline 0 & $\frac{\pi}{2}$ & $\left\{\frac{\pi}{2}\right\}$ & 4 & $\frac{3 \pi}{8}$ & $\left\{\frac{\pi}{2}, 0, \frac{\pi}{4},-\frac{\pi}{4}, \frac{3 \pi}{8}\right\}$ \\
1 & 0 & $\left\{\frac{\pi}{2}, 0\right\}$ & 5 & $\frac{\pi}{8}$ & $\left\{\frac{\pi}{2}, 0, \frac{\pi}{4},-\frac{\pi}{4}, \frac{3 \pi}{8}, \frac{\pi}{8}\right\}$ \\
2 & $\frac{\pi}{4}$ & $\left\{\frac{\pi}{2}, 0, \frac{\pi}{4}\right\}$ & 6 & $-\frac{\pi}{8}$ & $\left\{\frac{\pi}{2}, 0, \frac{\pi}{4},-\frac{\pi}{4}, \frac{3 \pi}{8}, \frac{\pi}{8},-\frac{\pi}{8}\right\}$ \\
3 & $-\frac{\pi}{4}$ & $\left\{\frac{\pi}{2}, 0, \frac{\pi}{4},-\frac{\pi}{4}\right\}$ & 7 & $-\frac{3 \pi}{8}$ & $\left\{\frac{\pi}{2}, 0, \frac{\pi}{4},-\frac{\pi}{4}, \frac{3 \pi}{8}, \frac{\pi}{8},-\frac{\pi}{8},-\frac{3 \pi}{8}\right\}$
\end{tabular}

Table 1: Parameter sets of the form (17). 


$$
\begin{aligned}
& R_{0}^{0}=1 \\
& R_{0}^{1}=z_{2} e_{2} \quad R_{1}^{1}=z_{1} e_{1} \\
& R_{0}^{2}=-z_{2}^{2} \quad R_{1}^{2}=-z_{1}^{2} \quad R_{2}^{2}=-\frac{1}{2}\left(z_{1}+z_{2}\right)^{2} \\
& R_{0}^{3}=-z_{2}^{3} e_{2} \quad R_{1}^{3}=-z_{1}^{3} e_{1} \quad R_{2}^{3}=-\frac{1}{4}\left(z_{1}+z_{2}\right)^{3}\left(e_{1}+e_{2}\right) \quad R_{3}^{3}=-\frac{1}{4}\left(z_{1}-z_{2}\right)^{3}\left(e_{1}-e_{2}\right)
\end{aligned}
$$

Table 2: First pseudo-complex powers associated with (17).

\section{A new combinatorial identity and the bijection between two types of Appell polynomials}

In this section we express the polynomials $\boldsymbol{P}_{k}\left(z_{1}, z_{2}\right)$ in terms of the basis $\left\{R_{s}^{k}\right\}_{s=0}^{k}$ with the parameter set given by (17). Table 3 contains the coordinates of the first $\boldsymbol{P}_{k}$ in terms of the referred basis. These results suggest that the special form of the polynomials $\boldsymbol{P}_{k}$ associated with the block structure of (17a), and consequently of $\left\{R_{0}^{k}, R_{1}^{k}, \ldots, R_{k}^{k}\right\}$, leads to an interesting representation of the standard Appell polynomials in terms of pseudo-complex powers.

\begin{tabular}{ll|ll}
$k$ & Coordinates of $\boldsymbol{P}_{k}$ & $k$ & Coordinates of $\boldsymbol{P}_{k}$ \\
\hline 0 & $(1)$ & 4 & $\left(\frac{1}{4}, \frac{1}{4}, \frac{1}{4}, \frac{1}{4}, 0\right)$ \\
1 & $\left(\frac{1}{2}, \frac{1}{2}\right)$ & 5 & $\left(\frac{1}{4}, \frac{1}{4}, \frac{1}{4}, \frac{1}{4}, 0,0\right)$ \\
2 & $\left(\frac{1}{2}, \frac{1}{2}, 0\right)$ & 6 & $\left(\frac{1}{4}, \frac{1}{4}, \frac{1}{4}, \frac{1}{4}, 0,0,0\right)$ \\
3 & $\left(\frac{1}{4}, \frac{1}{4}, \frac{1}{4}, \frac{1}{4}\right)$ & 7 & $\left(\frac{1}{8}, \frac{1}{8}, \frac{1}{8}, \frac{1}{8}, \frac{1}{8}, \frac{1}{8}, \frac{1}{8}, \frac{1}{8}\right)$
\end{tabular}

Table 3: Coordinates of $\boldsymbol{P}_{k}$ in the basis $\left\{R_{s}^{k}\right\}_{s=0}^{k}$.

Previously to the main theorems we prove two results of own interest. The first proposition fills in a gap in the explicit expression of the coefficients of the standard Appell polynomials (8), while the second one presents auxiliary relations deduced from properties of primitive roots of unity.

Proposition 1 Let $\left[z_{1}^{k-s} \times z_{2}^{s}\right]_{\boldsymbol{P}}$ denote the coefficient of $\boldsymbol{P}_{k}$ associated with the generalized power $z_{1}^{k-s} \times z_{2}^{s}$, for each $k=0,1, \ldots$ and $s=0,1, \ldots, k$.

I. If $k$ is even then

$$
\left[z_{1}^{k-s} \times z_{2}^{s}\right]_{\boldsymbol{P}}= \begin{cases}(-1)^{\frac{k}{2}} c_{k}\left(\begin{array}{l}
\frac{k}{2} \\
\frac{s}{2}
\end{array}\right), & \text { if s even } \\
0, & \text { if s odd }\end{cases}
$$

II. If $k$ is odd then

$$
\left[z_{1}^{k-s} \times z_{2}^{s}\right]_{\boldsymbol{P}}= \begin{cases}c_{k}(-1)^{\frac{k-1}{2}}\left(\begin{array}{c}
\frac{k-1}{2} \\
\frac{s}{2}
\end{array}\right) e_{1}, & \text { if } s \text { even } \\
c_{k}(-1)^{\frac{k-1}{2}}\left(\begin{array}{c}
\frac{k-1}{2} \\
\frac{s-1}{2}
\end{array}\right) e_{2}, & \text { if } s \text { odd } .\end{cases}
$$

Proof. Consider the polynomial $F_{k}(\lambda)$ of degree $k$ in the real parameter $\lambda$,

$$
F_{k}(\lambda)=\left(e_{1}+\lambda e_{2}\right)^{k}=\sum_{s=0}^{k}\left(\begin{array}{l}
k \\
s
\end{array}\right) \lambda^{s} e_{1}^{k-s} \times e_{2}^{s}
$$

and note that $F_{k}^{(s)}(0)=s !\left(\begin{array}{c}k \\ s\end{array}\right) e_{1}^{k-s} \times e_{2}^{s}$. Then, based on (8) we have

$$
\left[z_{1}^{k-s} \times z_{2}^{s}\right]_{\boldsymbol{P}}=\frac{c_{k}}{s !} F_{k}^{(s)}(0),
$$


which means that the expected result can be deduced by direct inspection of $F_{k}^{(s)}(0)$.

We observe that $F_{k}(\lambda)$ is a power of a pure vector and therefore

$$
F_{k}(\lambda)= \begin{cases}\left(-1-\lambda^{2}\right)^{\frac{k}{2}}, & \text { if } \quad k \text { even; } \\ \left(-1-\lambda^{2}\right)^{\frac{k-1}{2}}\left(e_{1}+\lambda e_{2}\right), & \text { if } \quad k \text { odd. }\end{cases}
$$

In order to obtain the higher order derivatives of $F_{k}$ we make use of a formula of $T$. Abadie which is an early version of Faà di Bruno's formula for the derivative of a composite function (see [28] and [29]). It reads as follows:

If $f$ and $g$ are real functions of $\lambda$, with a sufficient number of derivatives, then

$$
(g \circ f)^{(s)}(\lambda)=\sum_{l=0}^{s}\left(\begin{array}{l}
s \\
l
\end{array}\right) g^{(l)}(f(\lambda))\left\{\frac{d^{s-l}}{d h^{s-l}}\left(\Delta_{h} f(\lambda)\right)^{l}\right\}_{h=0},
$$

where $\Delta_{h} f(\lambda):=\frac{f(\lambda+h)-f(\lambda)}{h}$ is the difference quotient of $f$.

We proceed now with the proof, considering the two cases corresponding to the parity of $k$.

I. $k$ even

We observe that $F_{k}(\lambda)$ can be written as $F_{k}(\lambda)=(g \circ f)(\lambda)$, with $g(\lambda)=(-1-\lambda)^{\frac{k}{2}}$ and $f(\lambda)=\lambda^{2}$. Since

and

$$
g^{(l)}(\lambda)=\frac{\frac{k}{2} !}{\left(\frac{k}{2}-l\right) !}(-1)^{l}(-1-\lambda)^{\frac{k}{2}-l}, \quad l=0,1, \ldots, \frac{k}{2},
$$

$$
\frac{d^{s-l}}{d h^{s-l}}\left(\Delta_{h} f(\lambda)\right)^{l}= \begin{cases}\frac{l !}{(2 l-s) !}(2 \lambda+h)^{2 l-s}, & \text { for } s-l \leq l \\ 0, & \text { otherwise, }\end{cases}
$$

formula (23) results in

$$
\begin{aligned}
F_{k}^{(s)}(\lambda) & =\sum_{l \geq \frac{s}{2}}^{s}\left(\begin{array}{l}
s \\
l
\end{array}\right) \frac{\left(\frac{k}{2}\right) !}{\left(\frac{k}{2}-l\right) !}(-1)^{l}\left(-1-\lambda^{2}\right)^{\frac{k}{2}-l}\left\{\frac{l !}{(2 l-s) !}(2 \lambda+h)^{2 l-s}\right\}_{h=0} \\
& =\sum_{l \geq \frac{s}{2}}^{s} \frac{s !}{(s-l) !} \frac{\left(\frac{k}{2}\right) !}{\left(\frac{k}{2}-l\right) !}(-1)^{l}\left(-1-\lambda^{2}\right)^{\frac{k}{2}-l}(2 \lambda)^{2 l-s}
\end{aligned}
$$

Therefore

$$
F_{k}^{(s)}(0)=\left\{\begin{array}{lll}
(-1)^{\frac{k}{2}} s !\left(\begin{array}{l}
\frac{k}{2} \\
\frac{s}{2}
\end{array}\right), & s & \text { even; } \\
0, & s & \text { odd }
\end{array}\right.
$$

and from (22), the final result follows.

II. $k$ odd

The relation $F_{k}(\lambda)=F_{k-1}(\lambda)\left(e_{1}+\lambda e_{2}\right)$ together with Leibniz's differentiation rule leads to

$$
F_{k}^{(s)}(0)=F_{k-1}^{(s)}(0) e_{1}+F_{k-1}^{(s-1)}(0) e_{2} .
$$

The final result (21) can now easily be deduced from the even case.

Proposition 2 If $N$ and $p$ are integers such that $N \geq 0$ and $p \neq k N$, for odd $k$, then the following equalities are true:

$$
\sum_{l=1}^{2 N}(-1)^{l} \cos \left(l p \frac{\pi}{N}\right)=\sum_{l=1}^{2 N}(-1)^{l} \sin \left(l p \frac{\pi}{N}\right)=0 .
$$


Proof. Let $\omega=\cos \left(\frac{\pi}{N}\right)+i \sin \left(\frac{\pi}{N}\right)$ be a $2 N$-th primitive root of unity. Since $\omega \neq 1$ we have

$$
\sum_{l=1}^{2 N} \omega^{l}=0
$$

We note that the assumption $p \neq k N$, for odd $k$, is equivalent to assume that $\omega^{p} \neq-1$. In this way, replacing $\omega$ by $-\omega^{p}$ in formula (24), we obtain

$$
\sum_{l=1}^{2 N}\left(-\omega^{p}\right)^{l}=0
$$

or equivalently,

$$
\sum_{l=1}^{2 N}(-1)^{l} \cos \left(l p \frac{\pi}{N}\right)+i \sum_{l=1}^{2 N}(-1)^{l} \sin \left(l p \frac{\pi}{N}\right)=0 .
$$

The next theorem refines the central binomial coefficient identity (3) proved by J. Riordan in [20] and recently studied by A. Sofo ([18]), as already mentioned in the Introduction.

Theorem 2 (Riordan-Sofo type identity) If $m$ is a positive integer and $s=0,1, \ldots, m$, then

$$
\sum_{u=0}^{s}(-1)^{s-u}\left(\begin{array}{l}
s \\
u
\end{array}\right) c_{2(m-u)}=\left(\begin{array}{c}
m \\
s
\end{array}\right)\left(\begin{array}{c}
2 m \\
2 s
\end{array}\right)^{-1} c_{2 m}
$$

Proof. The function

$$
S_{m}(s):=\sum_{u=0}^{s}(-1)^{s-u}\left(\begin{array}{l}
s \\
u
\end{array}\right) c_{2(m-u)}
$$

can be written, for $s=1,2, \ldots, m$, as

$$
S_{m}(s)=\sum_{u=0}^{s-1}(-1)^{s-u}\left(\begin{array}{c}
s-1 \\
u
\end{array}\right) c_{2(m-u)}+\sum_{u=0}^{s-1}(-1)^{s-u}\left(\begin{array}{c}
s-1 \\
u
\end{array}\right) c_{2(m-1-u)}
$$

and therefore verifies the recurrence relation

$$
\begin{aligned}
& S_{m}(s)=-S_{m}(s-1)+S_{m-1}(s-1), \text { for } s \geq 1 ; \\
& S_{m}(0)=c_{2 m} .
\end{aligned}
$$

Since the weighted central binomial coefficients $c_{k}$ satisfy

$$
c_{2 m}=\frac{2 m-1}{2 m} c_{2 m-2},
$$

(cf. (1)) we can write

$$
\begin{aligned}
S_{m}(1) & =\frac{1}{2 m-1} c_{2 m} ; \\
S_{m}(2) & =\frac{3}{2 m-3} S_{m}(1)=\frac{1}{2 m-1} \frac{3}{2 m-3} c_{2 m} ; \\
\quad \cdots & \\
S_{m}(s) & =\frac{2 s-1}{2 m-(2 s-1)} S_{m}(s-1)=\frac{1}{2 m-1} \frac{3}{2 m-3} \cdots \frac{2 s-1}{2 m-(2 s-1)} c_{2 m} .
\end{aligned}
$$

Using now induction on $s$ we can prove that $S_{m}(s)=d(m, s) c_{2 m}$, where 


$$
d(m, s):=\prod_{l=0}^{s-1} \frac{2 l+1}{2 m-(2 l+1)}=\frac{(2 s-1) ! !(2(m-s)-1) ! !}{(2 m-1) ! !} .
$$

Finally, recalling relation $(2 k-1) ! !=\frac{(2 k) !}{2^{k} k !}$, we obtain

$$
d(m, s)=\frac{m !(2 m-2 s) !(2 s) !}{(m-s) ! s !(2 m) !}=\left(\begin{array}{c}
m \\
s
\end{array}\right)\left(\begin{array}{c}
2 m \\
2 s
\end{array}\right)^{-1}
$$

and the desired result follows.

Remark 2 If $s=m$, then relation (25) reduces to the identity (3), thus justifying its designation as a Riordan-Sofo type binomial identity.

Finally, we are able to prove the main result of this paper concerning the relationship between two types of Appell polynomials in $\mathbb{R}^{3}$, namely (8) and a special case of (14).

Theorem 3 The standard Appell polynomials $\boldsymbol{P}_{k}$ can be written in terms of the pseudo-complex powers $R_{l}^{k}$ as

$$
\boldsymbol{P}_{k}\left(z_{1}, z_{2}\right)=\frac{1}{2^{n}} \sum_{l=0}^{2^{n}-1} R_{l}^{k}\left(z_{1}, z_{2}\right)
$$

where $n$ is the integer for which $2^{n}-1 \leq k \leq 2\left(2^{n}-1\right)$.

Proof. Denote by $\boldsymbol{R}_{k}\left(z_{1}, z_{2}\right)$ the right-hand side of (26). The proof consists in comparing $\left[z_{1}^{k-s} \times z_{2}^{s}\right]_{\boldsymbol{P}}$ with $\left[z_{1}^{k-s} \times z_{2}^{s}\right]_{\boldsymbol{R}}$, i.e. the coefficients of $z_{1}^{k-s} \times z_{2}^{s}$ of $\boldsymbol{P}_{k}$ with those of $\boldsymbol{R}_{k}$. We first note that due to (18) and (19) we can write

$$
\boldsymbol{R}_{k}\left(z_{1}, z_{2}\right)=\frac{1}{2^{n}} \sum_{l=-2^{n-1}+1}^{2^{n-1}}\left(z_{1} \cos \left(\frac{l \pi}{2^{n}}\right)+z_{2} \sin \left(\frac{l \pi}{2^{n}}\right)\right)^{k}\left(e_{1} \cos \left(\frac{l \pi}{2^{n}}\right)+e_{2} \sin \left(\frac{l \pi}{2^{n}}\right)\right)^{k}
$$

We continue the proof by considering again two cases, depending on the parity of $k$.

I. $k$ even

Since an even power of a pure vector is real valued, (27) simplifies for $k=2 m$ to

$$
\boldsymbol{R}_{2 m}\left(z_{1}, z_{2}\right)=\frac{(-1)^{m}}{2^{n}} \sum_{l=-2^{n-1}+1}^{2^{n-1}}\left(z_{1} \cos \left(\frac{l \pi}{2^{n}}\right)+z_{2} \sin \left(\frac{l \pi}{2^{n}}\right)\right)^{2 m}
$$

which means that only even powers of $z_{1}$ and $z_{2}$ are present in the last expression (cf. (5)) and therefore

$$
\boldsymbol{R}_{2 m}\left(z_{1}, z_{2}\right)=\frac{(-1)^{m}}{2^{n}}\left(z_{1}^{2 m}+z_{2}^{2 m}+2 \sum_{l=1}^{2^{n-1}-1} \sum_{s=0}^{m}\left(\begin{array}{c}
2 m \\
2 s
\end{array}\right)\left(\cos \left(\frac{l \pi}{2^{n}}\right)\right)^{2 m-2 s}\left(\sin \left(\frac{l \pi}{2^{n}}\right)\right)^{2 s} z_{1}^{2 m-2 s} \times z_{2}^{2 s}\right) .
$$

After simple manipulation and direct inspection we obtain

$$
\left[z_{1}^{2 m-2 s} \times z_{2}^{2 s}\right]_{\boldsymbol{R}}=\frac{(-1)^{m}}{2^{n}}\left(\begin{array}{c}
2 m \\
2 s
\end{array}\right) \tilde{S}_{m}(s),
$$

where

$$
\tilde{S}_{m}(s):=\sum_{l=1}^{2^{n}}\left(\cos \left(\frac{l \pi}{2^{n}}\right)\right)^{2 m-2 s}\left(\sin \left(\frac{l \pi}{2^{n}}\right)\right)^{2 s} .
$$

This last sum can be written as

$$
\tilde{S}_{m}(s)=\sum_{l=1}^{2^{n}}\left(\cos \left(\frac{l \pi}{2^{n}}\right)\right)^{2 m-2 s}\left(1-\cos ^{2}\left(\frac{l \pi}{2^{n}}\right)\right)^{s}=\sum_{u=0}^{s}(-1)^{u}\left(\begin{array}{c}
s \\
u
\end{array}\right) \sum_{l=1}^{2^{n}}\left(\cos \left(\frac{l \pi}{2^{n}}\right)\right)^{2 m-2(s-u)} .
$$


Then we use the well known identity

$$
(\cos \theta)^{2 N}=\frac{1}{2^{2 N}}\left(\begin{array}{c}
2 N \\
N
\end{array}\right)+\frac{1}{2^{2 N-1}} \sum_{t=0}^{N-1}\left(\begin{array}{c}
2 N \\
t
\end{array}\right) \cos (2(N-t) \theta)
$$

written in terms of the weighted central binomial coefficients (2) as

$$
(\cos \theta)^{2 N}=c_{2 N}+\frac{1}{2^{2 N-1}} \sum_{t=0}^{N-1}\left(\begin{array}{c}
2 N \\
t
\end{array}\right) \cos (2(N-t) \theta)
$$

in order to obtain

$$
\tilde{S}_{m}(s)=\sum_{u=0}^{s}(-1)^{u}\left(\begin{array}{l}
s \\
u
\end{array}\right) 2^{n} c_{2(m-s+u)}+A(m, s)
$$

where

$$
A(m, s)=\sum_{u=0}^{s}\left(\begin{array}{l}
s \\
u
\end{array}\right) \frac{(-1)^{u}}{2^{2(m-s+u)-1}} \sum_{t=0}^{m-s+u-1}\left(\begin{array}{c}
2(m-s+u) \\
t
\end{array}\right) \sum_{l=1}^{2^{n}} \cos \left(2(m-s+u-t) \frac{l \pi}{2^{n}}\right) .
$$

Since $2^{n}-1 \leq 2 m \leq 2\left(2^{n}-1\right)$ we can write $2 m=2^{n}+2 m^{\prime}$, for $0 \leq m^{\prime} \leq 2^{n-1}-1$ and conclude that

$$
\sum_{l=1}^{2^{n}} \cos \left(2(m-s+u-t) \frac{l \pi}{2^{n}}\right)=\sum_{l=1}^{2^{n}}(-1)^{l} \cos \left(\left(m^{\prime}-s+u-t\right) \frac{l \pi}{2^{n-1}}\right) .
$$

Now, in order to be able to apply Proposition 2, we need to ensure that $m^{\prime}-s+u-t$ is not an odd multiple of $2^{n-1}$. Based on the fact that $0 \leq t \leq m-s+u-1$ and $u-s \leq 0$, we are led to the relation

$$
m^{\prime}-m+1=-2^{n-1}+1 \leq m^{\prime}-s+u-t \leq m^{\prime} \leq 2^{n-1}-1
$$

and consequently, by the use of Proposition 2, the aforementioned sum vanishes and therefore also the sum $A(m, s)$ in (30). Finally, comparing now (30) with the the Riordon-Sofo type identity (25), we see that

$$
\tilde{S}_{m}(s)=\sum_{u=0}^{s}(-1)^{u}\left(\begin{array}{l}
s \\
u
\end{array}\right) 2^{n} c_{2(m-s+u)}=2^{n}\left(\begin{array}{c}
m \\
s
\end{array}\right)\left(\begin{array}{c}
2 m \\
2 s
\end{array}\right)^{-1} c_{2 m} .
$$

Replacing $\tilde{S}_{m}(s)$ in (28) and comparing with (20) we obtain

$$
\left[z_{1}^{2 m-2 s} \times z_{2}^{2 s}\right]_{\boldsymbol{R}}=(-1)^{m}\left(\begin{array}{c}
m \\
s
\end{array}\right) c_{2 m}=\left[z_{1}^{2 m-2 s} \times z_{2}^{2 s}\right]_{\boldsymbol{P}}
$$

and the even case is proved.

II. $k$ odd

If $k$ is of the form $k=2 m-1$ then (27) simplifies to

$$
\begin{aligned}
\boldsymbol{R}_{2 m-1}\left(z_{1}, z_{2}\right)= & \frac{(-1)^{m-1}}{2^{n}} \sum_{l=-2^{n-1}+1}^{2^{n-1}}\left(z_{1} \cos \left(\frac{l \pi}{2^{n}}\right)+z_{2} \sin \left(\frac{l \pi}{2^{n}}\right)\right)^{2 m-1}\left(e_{1} \cos \left(\frac{l \pi}{2^{n}}\right)+e_{2} \sin \left(\frac{l \pi}{2^{n}}\right)\right) \\
= & \frac{(-1)^{m-1}}{2^{n}} \sum_{l=1}^{2^{n-1}}\left(z_{1} \cos \left(\frac{l \pi}{2^{n}}\right)+z_{2} \sin \left(\frac{l \pi}{2^{n}}\right)\right)^{2 m-1}\left(e_{1} \cos \left(\frac{l \pi}{2^{n}}\right)+e_{2} \sin \left(\frac{l \pi}{2^{n}}\right)\right) \\
& +\frac{(-1)^{m-1}}{2^{n}} \sum_{l=0}^{2^{n-1}-1}\left(z_{1} \cos \left(\frac{l \pi}{2^{n}}\right)-z_{2} \sin \left(\frac{l \pi}{2^{n}}\right)\right)^{2 m-1}\left(e_{1} \cos \left(\frac{l \pi}{2^{n}}\right)-e_{2} \sin \left(\frac{l \pi}{2^{n}}\right)\right)
\end{aligned}
$$


Developing the last expression in terms of symmetric powers (see (5)) we obtain

$$
\left[z_{1}^{2 m-1-2 s} \times z_{2}^{2 s}\right]_{\boldsymbol{R}}=\frac{(-1)^{m-1}}{2^{n}}\left(\begin{array}{c}
2 m-1 \\
2 s
\end{array}\right) \tilde{S}_{m}(s) e_{1}
$$

and

$$
\left[z_{1}^{2 m-2(s+1)} \times z_{2}^{2 s+1}\right]_{\boldsymbol{R}}=\frac{(-1)^{m-1}}{2^{n}}\left(\begin{array}{c}
2 m-1 \\
2 s+1
\end{array}\right) \tilde{S}_{m}(s+1) e_{2},
$$

where $\tilde{S}_{m}$ is the sum (29). The result follows by using (31) and comparing with (21).

The block structure presented in Theorem 3 implies that, for each block, i.e. for $2^{n}-1 \leq k \leq 2\left(2^{n}-1\right)$, the number of pseudo-complex powers necessary for the expression of each standard Appell polynomial does not vary along the block and corresponds to $2^{n}$ (see Table 3). This means that the degree $k=2^{n}-1$ is the one for which only $k+1$ polynomials are needed. For all the other values of $k$ in the block, there is a considerable reduction of the number of basis elements, specially if we look at the final value $k=2\left(2^{n}-1\right)$, where only $\frac{k}{2}+1$ polynomials are used.

\section{Final remarks}

Appell polynomials can play an important role in applications, namely in 3D-mapping problems (see [30, 31]) or in the construction of classes of generalized classical polynomials (cf. [32, 33]).

In this work we consider a basis of 3D monogenic Appell polynomials isomorphic to the complex powers, which is particularly easy to handle, from the computational point of view. It is rather surprising that, using the parameter set (17), each standard Appell polynomial $\boldsymbol{P}_{k}$ is obtained as the arithmetic mean of $2^{n}$ polynomials $R_{l}^{k}$, for $k=2^{n}-1,2^{n}, \ldots, 2\left(2^{n}-1\right)$, with one and the same parameter-vectors. In addition, the use of (17) led to a link between combinatorics and hypercomplex function theory, by means of a new Riordan-Sofo type binomial identity.

Finally, we point out that, apart from the concrete parameter choice, the pseudo-complex polynomials $\mathcal{R}_{\boldsymbol{i}}^{k}$ obey all the same principle structure, isomorphic to the complex powers $z^{k}$. This fact allows to develop efficient algorithms for the construction of bases for the space of homogeneous monogenic paravector valued polynomials in $\mathbb{R}^{3}$. Here we concentrated on the structural aspects of pseudo-complex powers due to their relevance in combinatorics, but more details on the numerically and algorithmically benefit of pseudo-complex powers can be found in [34].

\section{Acknowledgments}

This work was supported by FEDER founds through COMPETE - Operational Programme Factors of Competitiveness ("Programa Operacional Factores de Competitividade") and by Portuguese funds through the Center for Research and Development in Mathematics and Applications (University of Aveiro) and the Portuguese Foundation for Science and Technology ("FCT - Fundação para a Ciência e a Tecnologia"), within project PEst-C/MAT/UI4106/2011 with COMPETE number FCOMP-01-0124-FEDER-022690. The research of the first author was also supported by FCT under the fellowship SFRH/BD/44999/2008.

\section{References}

[1] K. Weierstrass, Math. Werke, Bd. 2, p. 235, Berlin 1897.

[2] Gr. C. Moisil, N. Théodoresco, Fonctions holomorphes dans l'espace, Mathematica 5 (1931) 142-159.

[3] R. Fueter, Analytische Funktionen einer Quaternionenvariablen, Comment. Math. Helv. 4 (1932) 9-20.

[4] R. Fueter, Funktionentheorie im Hyperkomplexen. Lecture notes written and supplemented by E. Bareiss, Math. Inst. Univ. Zrich, Herbstsemester 1948/49.

[5] R. Delanghe, On regular-analytic functions with values in a Clifford algebra, Math. Ann. 185 (1970) 91-111. 
[6] F. Brackx, Two powerful theorems in Clifford analysis, AIP Conference Proceedings 1281 (2010) 3-7.

[7] F. Brackx, R. Delanghe, F. Sommen, Clifford Analysis, Pitman 76, London, 1982.

[8] K. Gürlebeck, K. Habetha, W. Sprössig, Holomorphic Functions in the Plane and n-Dimensional Space, Birkäuser Verlag, Basel, 2008.

[9] I. Cação, M. I. Falcão, H. R. Malonek, Matrix representations of a special polynomial sequence in arbitrary dimension, Computational Methods and Function Theory 12 (2) (2012) 371-391.

[10] M. I. Falcão, H. R. Malonek, Generalized exponentials through Appell sets in $\mathbb{R}^{n+1}$ and Bessel functions, AIP Conference Procedings 936 (2007) 738-741.

[11] M. I. Falcão, J. F. Cruz, H. R. Malonek, Remarks on the generation of monogenic functions, in: K. Gürlebeck and C. Könke (Ed.), 17th Inter. Conf. on the Appl. of Computer Science and Mathematics on Architecture and Civil Engineering, Weimar, (2006).

[12] R. Lávička, Canonical bases for $\mathfrak{s l}(2, \mathbb{C})$-modules of spherical monogenics in dimension 3 , Archivum Mathematicum 46 (2010) 339-349.

[13] Delanghe, R. and Lávička, R. and Souček, V., The Gelfand-Tsetlin bases for Hodge-de Rham systems in Euclidean spaces, Math. Methods Appl. Sci., 35 (7) (2012) 745-757.

[14] S. Bock, K. Gürlebeck, On a generalized Appell system and monogenic power series, Math. Methods Appl. Sci. 33 (4) (2010) 394-411.

[15] H. R. Malonek, M. I. Falcão, 3D-mappings using monogenic functions, In: Th. E. Simos et al. (Ed.) ICNAAM-2006 Conference Proceedings, Wiley-VCH, Weinheim, (2006) 615-619.

[16] I. Cação, H. R. Malonek, On complete sets of hypercomplex Appell polynomials, AIP Conference Proceedings 1048 (2008) 647-650.

[17] H. D. Lehmer, Interesting Series Involving the Central Binomial Coefficient, Amer. Math. Monthly 92 (1985) 449-457.

[18] A. Sofo, Closed form representation of binomial sums and series, Le Matematiche vol. LIV (1999) 175-186.

[19] A. Sofo, Computational Techniques for the Summation of Series, Kluwer, New York, 2003.

[20] J. Riordan, Combinatorial Identities, John Wiley \& Sons Inc., New York, 1968.

[21] H. Prodinger, Knuth's old sum - a survey, European Association for Theoretical Computer Science Bulletin 54 (1994) 232-245.

[22] M. I. Falcão, H. R. Malonek, On paravector valued homogeneous monogenic polynomials with binomial expansion, Adv. in Appl. Clifford Algebras, 22 (3) (2012) 789-801.

[23] K. Gürlebeck, H. R. Malonek, A hypercomplex derivative of monogenic functions in $\mathbb{R}^{n+1}$ and its applications, Complex Variables Theory Appl. 39 (1999) 199-228.

[24] H. R. Malonek, Selected topics in hypercomplex function theory, in: S.-L. Eriksson (Ed.), Clifford Algebras and Potential Theory, University of Joensuu 7 (2004) 111-150.

[25] H. R. Malonek, Power series representation for monogenic functions in $\mathbb{R}^{n+1}$ based on a permutational product, Complex Variables, Theory Appl. 15 (1990) 181-191.

[26] S. G. Krantz, Function Theory of Several Complex Variables, 2nd ed., American Mathematical Society, Providenc, RI, 2001.

[27] P. Appell, Sur une classe de pôlynomes, Annals scientifiques de l'École Normal Supérieure 21 série 9 (2) (1880) 119-144. 
[28] W. P. Johnson, The curious history of Faà di Bruno's formula, The Mathematical Association of America Monthly 109 (3) (2002) 217-234.

[29] C. F. Faà di Bruno, Note sur une nouvelle formule de calcul différentiel, Quarterly J. Pure Appl. Math. 1 (1857) 359-360.

[30] H. R. Malonek, R. de Almeida, A note on a generalized Joukowski transformation, Appl. Math. Lett. 23 (2010), no. 10, 1174-1178.

[31] C. Cruz, M. I. Falcão, H. R. Malonek, 3D Mappings by Generalized Joukowski Transformation. Lecture Notes in Computer Science, 6784,(2011) 358-373.

[32] I. Cação, M. I. Falcão, H. R. Malonek, Laguerre derivative and monogenic Laguerre polynomials: An operational approach, Math. Comput. Model. 53 (5-6) (2011) 1084-1094.

[33] I. Cação, M. I. Falcão, H. R. Malonek, On generalized hypercomplex Laguerre-type exponentials and applications, . Lecture Notes in Computer Science, 6784,(2011) 271-286.

[34] C. Cruz, M. I. Falcão, H. R. Malonek, On pseudo-complex bases for monogenic polynomials. AIP Conference Proceedings 1493 (2012) 350-355 